\title{
A!
}

This is an electronic reprint of the original article.

This reprint may differ from the original in pagination and typographic detail.

Montoya Moreno, Resti; Ala-Laurinaho, Juha; Viikari, Ville

\section{Rod Waveguides as Future 5G Antennas for Mobile Devices}

Published in:

Proceedings of the 48th European Microwave Conference

DOI:

10.23919/EuMC.2018.8541770

Published: 01/01/2018

Document Version

Peer reviewed version

Please cite the original version:

Montoya Moreno, R., Ala-Laurinaho, J., \& Viikari, V. (2018). Rod Waveguides as Future 5G Antennas for Mobile Devices. In Proceedings of the 48th European Microwave Conference (pp. 1081-1084). (European Microwave Conference). IEEE. https://doi.org/10.23919/EuMC.2018.8541770

This material is protected by copyright and other intellectual property rights, and duplication or sale of all or part of any of the repository collections is not permitted, except that material may be duplicated by you for your research use or educational purposes in electronic or print form. You must obtain permission for any other use. Electronic or print copies may not be offered, whether for sale or otherwise to anyone who is not an authorised user. 
This is the accepted version of the original article published by IEEE.

(C) 2018 IEEE. Personal use of this material is permitted. Permission from IEEE must be obtained for all other uses, in any current or future media, including reprinting/republishing this material for advertising or promotional purposes, creating new collective works, for resale or redistribution to servers or lists, or reuse of any copyrighted component of this work in other works. 


\title{
Rod Waveguides as Future 5G Antennas for Mobile Devices
}

\author{
Resti Montoya Moreno, Juha Ala-Laurinaho, Ville Viikari \\ Aalto University School of Electrical Engineering, Espoo, Finland, resti.montoyamoreno@aalto.fi
}

\begin{abstract}
This article describes a novel dual polarized rod waveguide antenna array for millimeter-wave frequencies. The antenna is fed from a flexible PCB which includes two orthogonal probes acting as feeding elements for the rod waveguide. Simulation results show that this antenna is a feasible solution that could be easily integrated inside a portable device to realize an end-fire beam steerable antenna array. According to simulations, a 4-element array placed inside a mobile phone provides higher than $65 \%$ efficiency at both polarizations across the whole band of 24-29.5 GHz. The matching level of a single element is better than $-10 \mathrm{~dB}$ and the active reflection coefficient is below $-6 \mathrm{~dB}$ in the whole band.

Keywords - antennas, mm-wave frequencies, 5G, rod waveguide.
\end{abstract}

\section{INTRODUCTION}

5G mobile communications networks are expected to provide enough capacity for the demands of machine to machine (M2M) communications, self-driving cars, augmented reality, and other emerging applications. $5 \mathrm{G}$ is not yet fully standardized, but the foreseen technical solutions to achieve high data rates include the uses of higher frequencies (6-300 GHz) and larger frequency bands. Among these higher frequency bands, 24 to $29.5 \mathrm{GHz}$ is being extensively studied and is one of the most interesting bands globally, and therefore we designed our antenna for this frequency range [1],[2]. The use of higher frequencies has one direct consequence which is the increase in free-space attenuation. This means that high gain antennas are necessary in order to compensate these losses. Moreover, since mobile phone terminals are in movement, beam steering capabilities are required.

Broad-side mm-wave antenna arrays are well known and present a lower degree of complexity when compared to end-fire antenna arrays [3],[4]. Current mobile phone antennas are typically based on capactive coupling elements (CCE). These antennas can be implemented by using the edge metal rim of the phone, which is the reason why millimeter-wave antennas can easily affect the operation and behavior of conventional antennas. However, the proposed rod waveguide antenna does not present a galvanic contact to the metal rim, keeping capacitive coupling low, and hence, reducing the effect on low band antennas.

Rod waveguides as antenna elements have been studied in the past; e.g. in [5]-[7]. However, this paper presents a novel feeding network consisting of a flexible PCB, which allows to feed dual-polarized operation from a single PCB in a reduced volume, making integration inside a phone simpler. Integration of the antenna inside a realistic mobile phone structure is studied by simulations.

\section{Dual Polarized Rod WAVEguide ANTEnNA}

One dielectric rod waveguide antenna consists of a feeding probe on a dielectric-filled rectangular waveguide, transition from bound (metallic wall) waveguide into unbound (no metallic wall) waveguide which is then tapered into a radiating section as shown in Figure 4. The feeding probes are realized on a flexible PCB, which is inserted in a narrow slot on a metal-walled waveguide. There are two orthogonal probes to excite two linear polarization. Radiating parts consist of only dielectric to avoid grounding the metal rim, which is used by the low-frequency antennas. The antenna is designed using CST STUDIO software and the material used for the rod waveguide is PREPERM L900HF with $\epsilon_{r}=9$ and $\tan \delta=5 * 10^{-3}$ [8]. The antenna is formed by a flexible PCB acting as the feeding structure and a rod waveguide as the radiating element. Copper is used as the material for the feeding probes, ground plane, vias, and waveguide platings. The phone model consists of a metallic enclosure with two layers of gorilla glass $\left(\epsilon_{r}=6.6, \tan \delta=0.02\right)$ [9] on top and bottom, and a slot in the front side which is filled with low permittivity plastic $\left(\epsilon_{r}=2\right.$ and $\left.\tan \delta=5 * 10^{-3}\right)$ in order for the electromagnetic waves to be radiated (See figures 1 and 2).

\section{A. Feeding structure}

The feeding structure consists of a flexible PCB (Figure 3) that partially penetrates the initial part of the rod waveguide. The material used for the PCB is Rogers RT5880 with $\epsilon_{r}=2.2$ and $\tan \delta=5 * 10^{-3}$ [10]. Two grounded coplanar waveguide (GCPW) structures are implemented in the horizontal part of the PCB. These two GCPWs are then continued as coplanar waveguides (CPW) in the vertical plane and the signal line thickness and gap widths are adjusted in order to keep the same impedance of the transmission line. These CPWs end in two orthogonal probes which excite horizontal and vertical modes inside the rod waveguide. Vias are included in the PCB in order to prevent the leakage of energy from the necessary opening in the waveguide. Losses in the feeding structure have been estimated using a dual-transition structure and are around $1 \mathrm{~dB}$ for each polarization.

\section{B. Rod Waveguide}

The rod waveguide (Figure 4) can be divided into three sections. Standard squared waveguide, horn shaped waveguide and tapered dielectric. Standard rectangular waveguides do not support both vertical and horizontal modes at the same 


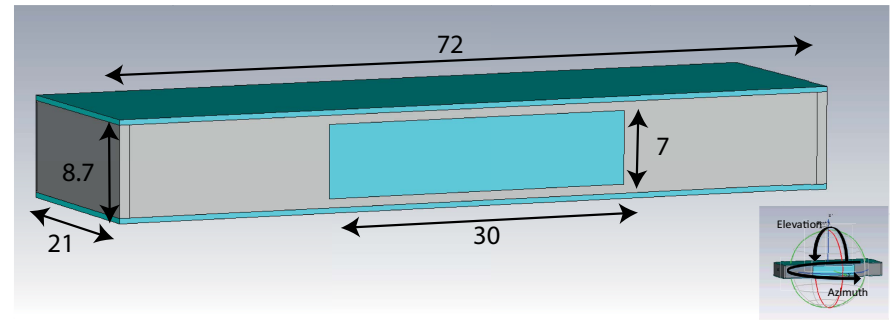

Fig. 1. Phone model and dimensions.

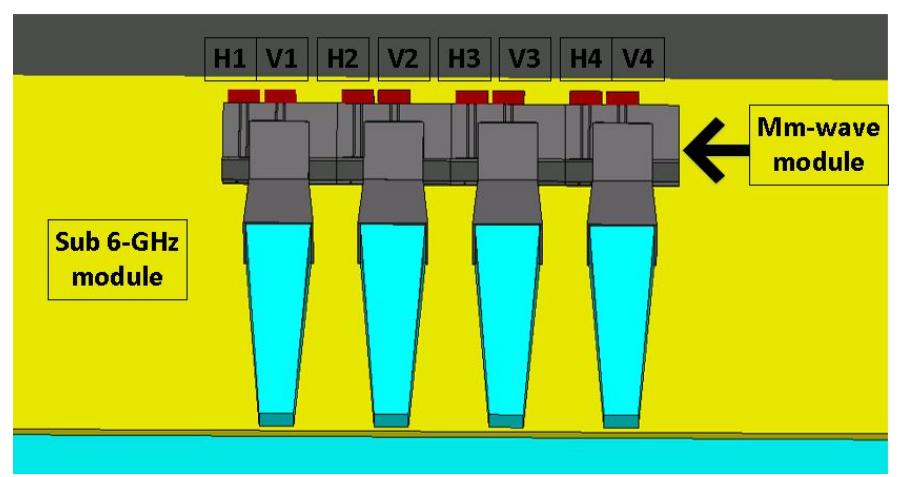

Fig. 2. Top view of rod waveguide array inside phone.

frequency, therefore a squared waveguide is necessary. The cutoff frequency of a waveguide is defined by :

$$
f_{c}=\frac{1}{2 \pi \sqrt{\epsilon \mu}} \sqrt{\left(\frac{m \pi}{a}\right)^{2}+\left(\frac{n \pi}{b}\right)^{2}}
$$

where $\epsilon$ is the permittivity, $\mu$ is the permeability, $a$ and $b$ are the waveguide dimensions, and $m$ and $n$ correspond to the order of propagated TE or TM mode [11].

Choosing the dimensions to be $2.4 \times 2.4 \mathrm{~mm}^{2}$ the cutoff frequency is found at $20.8 \mathrm{GHz}$ so it is ensured that the waveguide works properly at the desired 24 to $29.5 \mathrm{GHz}$ range.

The horn shaped part and the final flared section of the rod waveguide serve the same purpose: both these sections provide a gradual transition to match the impedance of the rod

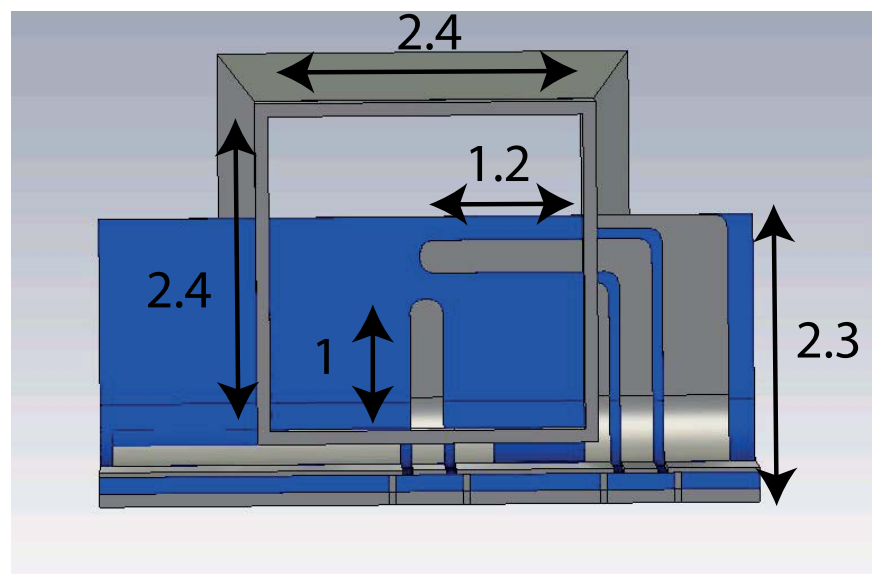

Fig. 3. Flexible PCB including two orthogonal probes in order to feed the rod waveguide.

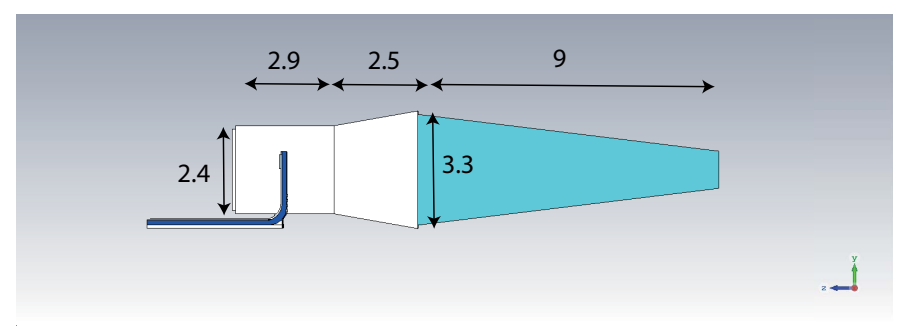

Fig. 4. Side view and dimensions of the rod waveguide in $\mathrm{mm}$.

waveguide filled with high permittivity plastic $\epsilon_{r}=9$ to free space. There are three possible types of tapering: pyramidal taper, E-plane taper and H-plane taper [5] .Pyramidal taper is considered for this design because of its symmetry with respect to both polarizations.

In the horn shaped section the maximum dimensions of the flare are $2.84 \times 3.27 \mathrm{~mm}^{2}$ and the total length is $2.5 \mathrm{~mm}$. The cross-section of the flare is not exactly squared due to the different feeding for vertical and horizontal polarizations, and in order to decrease the coupling between polarizations. The third section is $9 \mathrm{~mm}$ long and its minimum cross-section at the end is $1.5 \times 1.1 \mathrm{~mm}^{2}$. There is a trade off between dimensions and performance. Long tapered section and larger flare provides better electrical performance at the expense of increased size.

\section{RESULTS}

The results of a single antenna element in free space and four element array inside a phone are presented in this section.

\section{A. Single element performance}

Figure 5 presents the S-parameters for the single antenna element. Reflection coefficient is below $-10 \mathrm{~dB}$ for both polarizations for the whole frequency band while coupling between the two different polarizations is kept below $-15 \mathrm{~dB}$. Figure 6 shows that the efficiency is kept above $75 \%$ for both polarizations and the realized gain is always above $6 \mathrm{dBi}$ and peaks at $7.9 \mathrm{dBi}$. Figures 7 and 8 show the realized gain values in azimuth and elevation planes, respectively, at 25, 27, and $29 \mathrm{GHz}$. The radiation pattern is almost invariant of frequency and cross polarization levels are kept below $-15 \mathrm{~dB}$ for the whole frequency range.

\section{B. Four element array performance}

Once the single element performance was optimized and analyzed a four element array is formed (Figure 2) and introduced inside a mobile phone like structure. Inter element spacing is set to $5 \mathrm{~mm}$ which corresponds to $\lambda / 2$ at $30 \mathrm{GHz}$. If the inter element spacing is kept below $\lambda / 2$ grating lobes are avoided when steering the beam [12].

The total width of the array is $20 \mathrm{~mm}$, while each element is around $17 \mathrm{~mm}$ long, making this four element array a very compact solution. Active reflection coefficients are shown in Figure 9, and these are kept below $-6 \mathrm{~dB}$ for the whole frequency band and mostly below $-10 \mathrm{~dB}$. Only the elements in the middle present slightly worse results at lower frequencies 


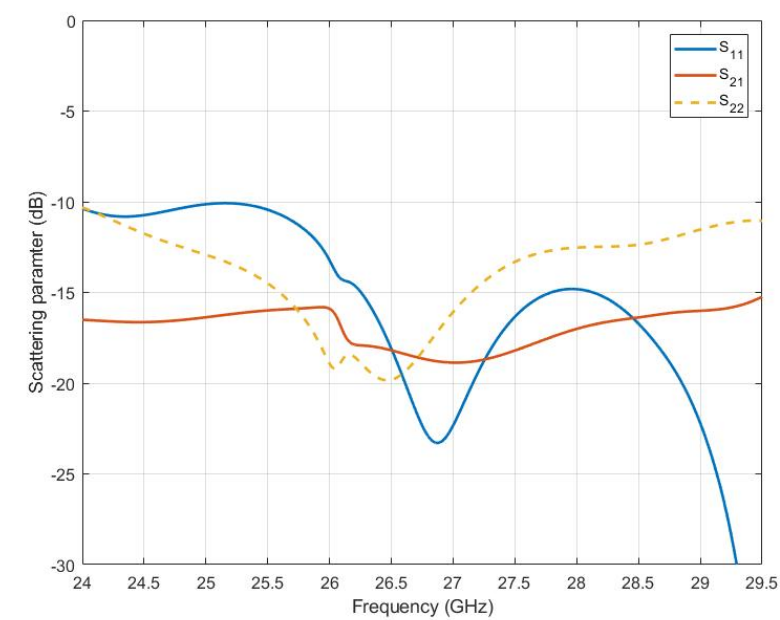

Fig. 5. Magnitudes of the scattering parameters for the single element.

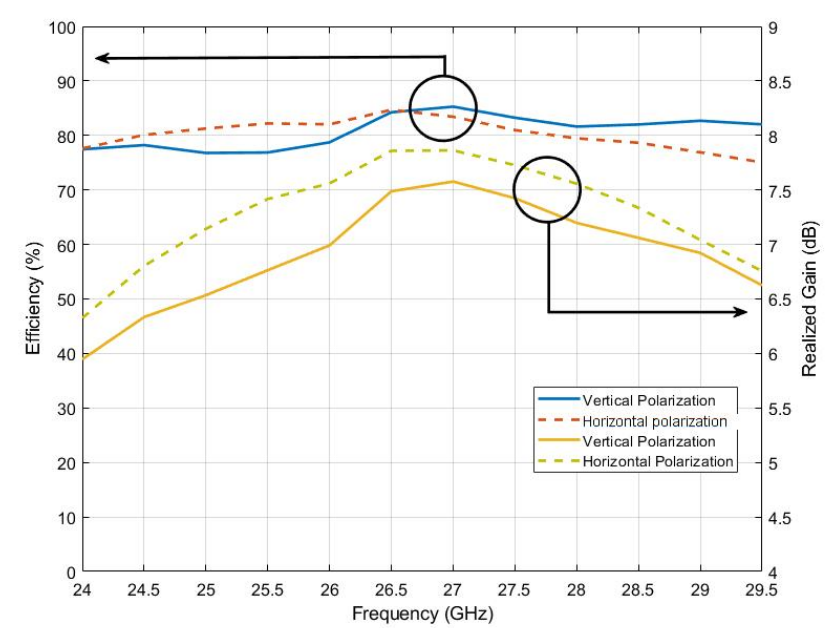

Fig. 6. Efficiency and realized gain of the single element.

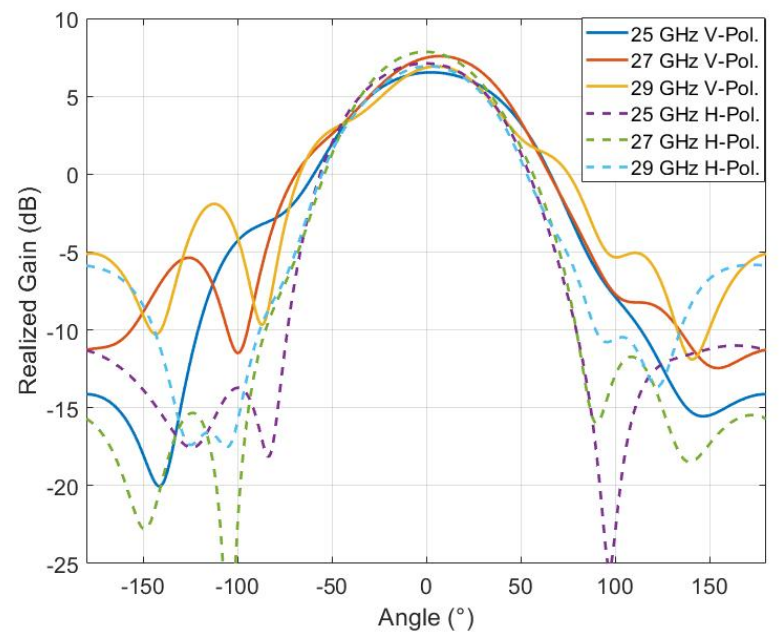

Fig. 7. Realized gain of the single element in the elevation plane.

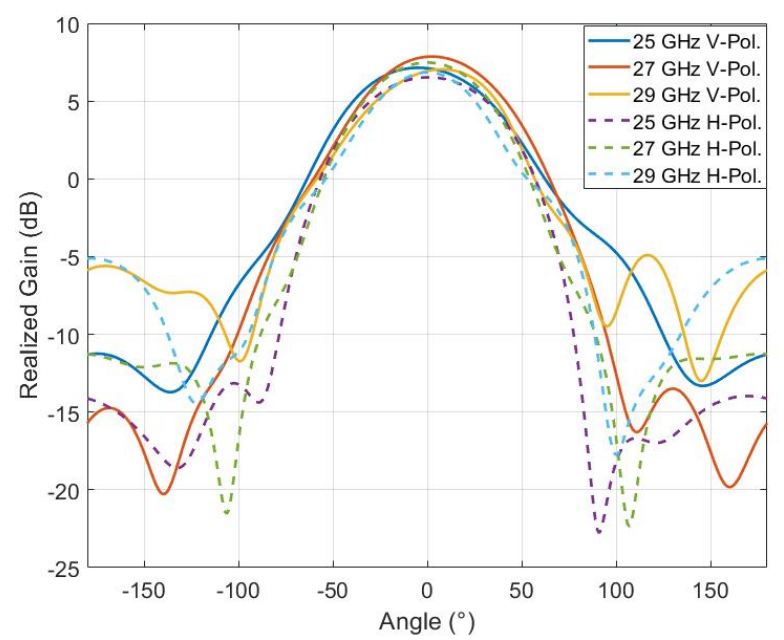

Fig. 8. Realized gain of the single element in the azimuth plane.

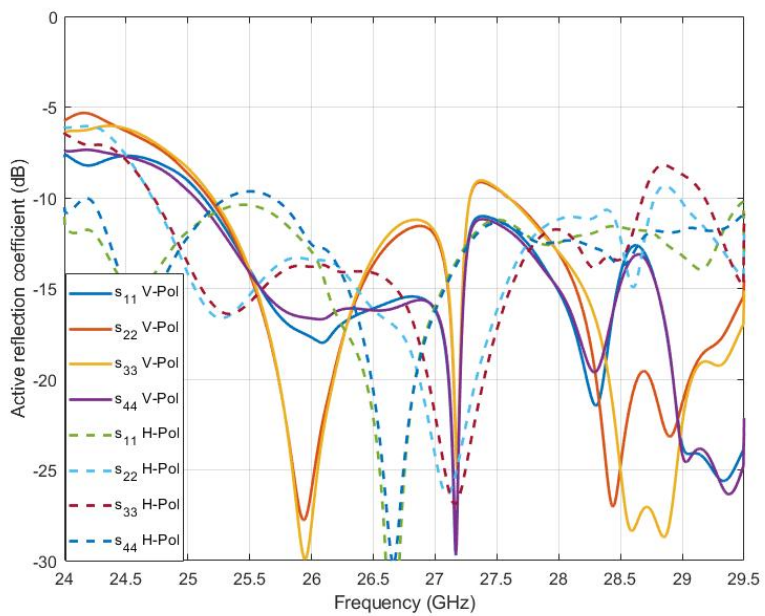

Fig. 9. Magnitudes of the active scattering parameters for the 4-element array.

due to the coupling from neighboring elements. Efficiency is above $65 \%$ for both polarizations as Figure 10 shows, and realized gain is above $9.5 \mathrm{dBi}$ peaking at $12.2 \mathrm{dBi}$.

Beam steering at $27 \mathrm{GHz}$ is presented in Figures 11 and 12. As these two figures show the beam can be steered up to $\pm 50^{\circ}$ with a scan loss below $3 \mathrm{~dB}$ for the vertical polarization and up to $\pm 34^{\circ}$ for the horizontal polarization. The beam steering range, which is restricted by the radiation pattern of one element, could be extended for the horizontal polarization by increasing the width of the slot in the frame of the mobile phone.

\section{CONCLUSION}

A novel dual polarized rod waveguide antenna array inside a mobile phone is presented in this paper. The antenna is designed to work at the $24-29.5 \mathrm{GHz}$ frequency range and by using high permittivity dielectric, the dimensions can be reduced making it a very suitable antenna for mobile devices. 


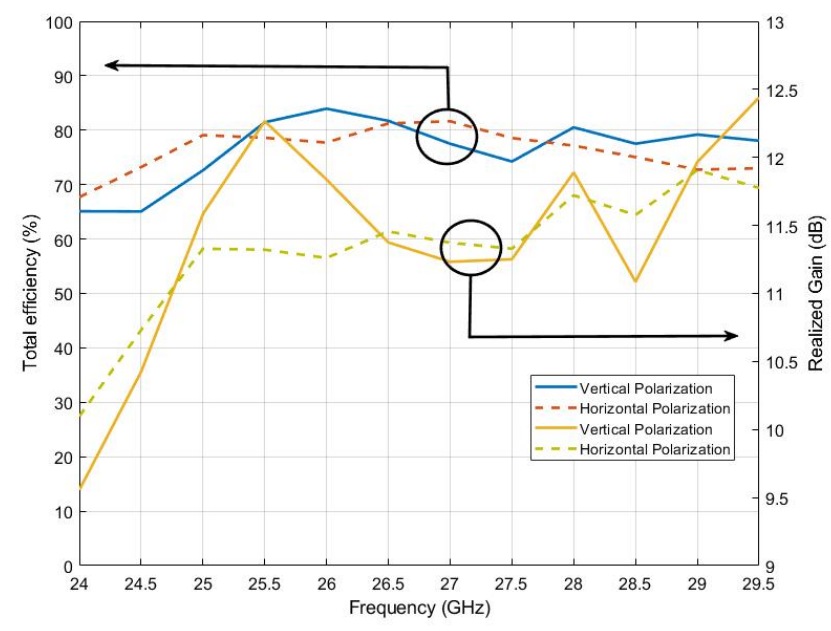

Fig. 10. Efficiency and realized gain of the 4-element array integrated with a mobile phone.

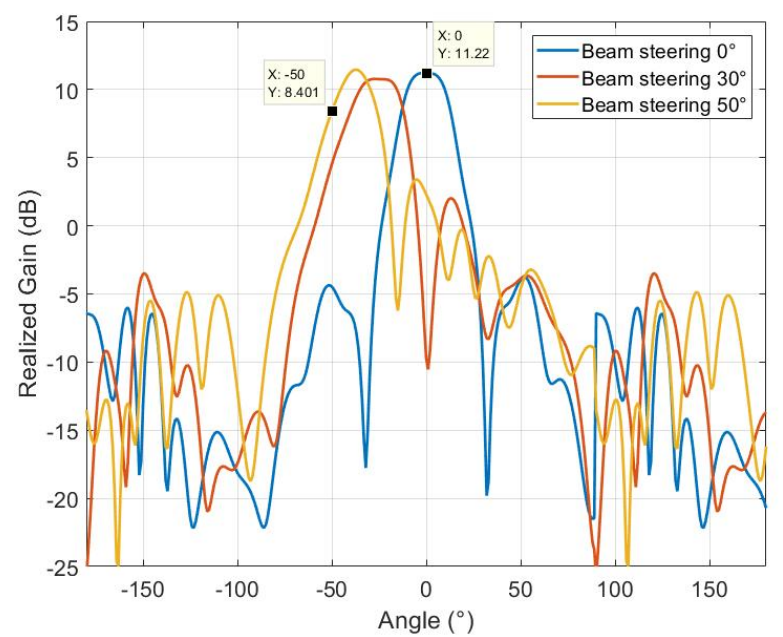

Fig. 11. Vertical polarization beam steering capabilities in the azimuth plane at $27 \mathrm{GHz}$.

Reflection coefficient for a single element is kept below $-10 \mathrm{~dB}$ for the whole band and active reflection coefficient is below $-6 \mathrm{~dB}$. Array efficiency is kept above $65 \%$ and peak gain is $12.2 \mathrm{dBi}$ for a 4-element array. The good performance verified by the simulation results and small dimensions make this antenna a very suitable solution for the upcoming 5th generation mobile communications.

\section{REFERENCES}

[1] S. Singh and P.Singh,"Key concepts and network architecture for 5G mobile technology," International Journal of Scientific Research Engineering \& Technology (IJSRET), vol. 1, no. 5, pp 165-170, Aug. 2012.

[2] GSMA Intelligence, "Understanding 5G: Perspectives on future technological advancements in mobile," London, UK, 2014.

[3] K. M. Morshed, K. P. Esselle, M. Heimlich, D. Habibi, and I. Ahmad, "Wideband slotted planar inverted-F antenna for millimeter wave 5G mobile devices," 10th Symposium on technologies for smart cities (TENSYMP), Bali, Indonesia, May 2016, pp 194-197).

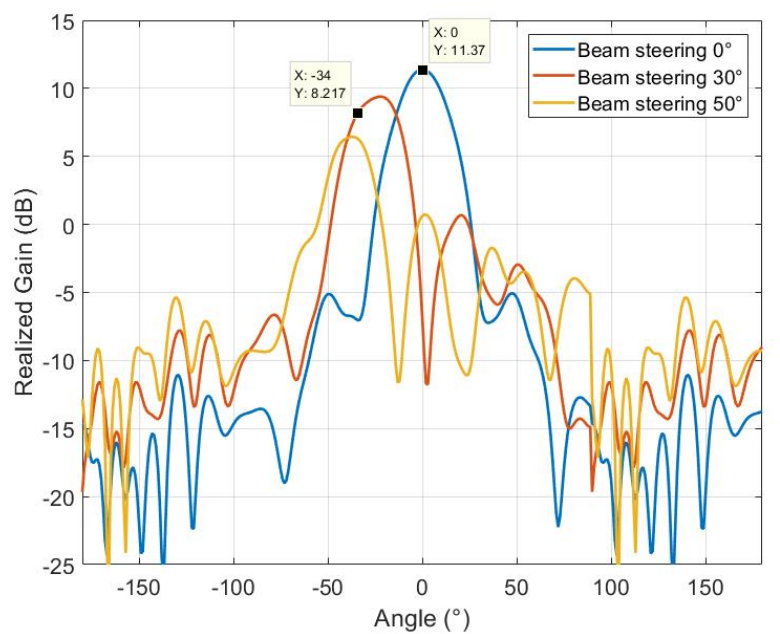

Fig. 12. Horizontal polarization beam steering capabilities in the azimuth plane at $27 \mathrm{GHz}$.

[4] A. T. Alreshaid, R. Hussain, S. K. Podilchak, and M. S. Sharawi, "A dual-element MIMO antenna system with a mm-wave antenna array,"10th European Conference on Antennas and Propagation (EuCAP), Davos, Switzerland, June 2016, pp. 1-4.

[5] A. Generalov, J. A. Haimakainen, D. V. Lioubtchenko, and A. V. Räisänen, "Wide band $\mathrm{mm}$ - and sub-mm-wave dielectric rod waveguide antenna," IEEE Trans. on Terahertz. Science and Technology, vol. 4, no. 5, pp. 568-574, Sept. 2014.

[6] J. P. Pousi, D. V. Lioubtchenko, S. N. Dudorov, and A. V. Räisänen, "High permittivity dielectric rod waveguide as an antenna array element for millimeter waves,'IEEE Trans. on Antennas and Propagation, vol. 58, no. 3 March 2010.

[7] A. A. Generalov, D. V. Lioubtchenko, and A. V. Räisänen,"Dielectric Rod Waveguide Antenna at 75-1100 GHz," 7th European Conference on Antennas and Propagation (EuCAP), Gothenburg, Sweden, April 2013, pp. 541-544.

[8] Premix Oy, Plastic Manufacturer. Helsinki, Finland, (accessed Oct. 2, 2017) [Online]. Available: https://www.preperm.com

[9] Corning Technologies, Gorilla Glass Manufacturer. New York, NY, USA, (accessed Oct. 10, 2017) [Online]. Available: https://www.corning.com

[10] Rogers Corporation, PCB Manufacturer. Chandler, AZ, USA, (accessed Oct. 3, 2017) [Online]. Available: https://www.rogerscorp.com/ documents/606/acs/RT-duroid-5870-5880-Data-Sheet.pdf

[11] D. M. Pozar, Microwave Engineering, 4th ed. Hoboken, NJ: John Wiley \& Sons, 2011.

[12] W. L. Stutzman and G. A. Thiele, Antenna Theory and Design, 3rd ed. Hoboken, NJ: John Wiley \& Sons, 2012. 